Published in final edited form as:

Can J Diabetes. 2017 August ; 41(4): 401-406. doi:10.1016/j.jcjd.2016.12.009.

\title{
Diabetes after Bariatric Surgery
}

\author{
Ankit Shah, $\mathrm{MD}^{\mathrm{a}}$ and Blandine Laferrère, $\mathrm{MD}, \mathrm{PhD}^{\mathrm{a}, \mathrm{b},{ }^{*}}$ \\ aDepartment of Medicine, Division of Endocrinology, College of Physicians and Surgeons, \\ Columbia University, New York, New York, United States

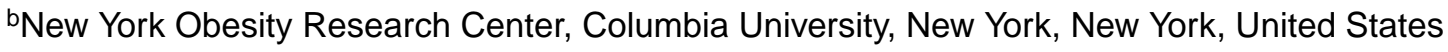

\begin{abstract}
Bariatric surgery has now emerged as an effective means of glycemic control in individuals with diabetes and obesity. However, long-term data show recurrence of hyperglycemia years after the procedure. Although the exact prevalence of diabetes relapse is unknown because of attrition and limited data on long-term follow up after the surgery, a significant percentage of patients experience relapse of diabetes. The mechanism of diabetes relapse is not completely understood and is not always linked to weight regain. The clinical implications of hyperglycemia after bariatric surgery for patients and healthcare providers is reviewed.
\end{abstract}

\section{Keywords}

bariatric surgery; glycemic control; relapse; remission; type 2 diabetes

\section{Introduction}

The prevalence of obesity and type 2 diabetes mellitus in North America and across the world has been increasing at alarming rates. Obesity is a critical risk factor for the development of type 2 diabetes. The relative risk for type 2 diabetes in individuals with obesity (body mass index [BMI] $\geq 30 \mathrm{~kg} / \mathrm{m}^{2}$ ) is 10 times greater than in those with normal BMIs ( $\geq 18.5$ to $\_4.9 \mathrm{~kg} / \mathrm{m}^{2}$ ). In addition, $90 \%$ of all individuals with type 2 diabetes are overweight or obese (1). Although intensive lifestyle modification, with diet-induced weight loss, exercise and intensive medical therapy can result in good control and even remission $(2,3)$ of type 2 diabetes, the majority of patients find it difficult to achieve sustained control of blood glucose. In addition, intensification of medical therapy can lead to hypoglycemia and weight gain.

Therefore, many have turned to bariatric surgery (BS) or metabolic surgery for treatment of obesity and type 2 diabetes. Surgical weight loss provides marked improvement in glycemic control, with the rate of type 2 diabetes remission varying from $24 \%$ to $95 \%$ at 2 years, depending on the type of surgery, the definition of remission and the type of subjects enrolled (2,4-6). The definition of remission varies from study to study but is generally

\footnotetext{
*Address for correspondence: Blandine Laferrère, MD, New York Obesity Research Centre, Columbia University, 1150 St. Nicholas Avenue R-121G, New York, New York 10032, United States., bbl14@ @olumbia.edu.
} 
defined as normoglycemia and glycated hemoglobin (A1C) levels below 6\%, without the need for glucose-lowering medications for at least 1 year (7). Recent long-term data, however, suggest that relapse of type 2 diabetes is common in the years following BS. Here we review clinical studies of type 2 diabetes relapse after BS and discuss pathophysiology and determinants of type 2 diabetes relapse and its clinical implications.

\section{Bariatric Surgery and Diabetes Remission}

Anecdotal evidence of improved glycemic control after gastrointestinal surgery in patients undergoing gastric resection for peptic ulcer disease or gastric cancer was reported as early as the first half of the 20th century (8). In the early 1980s, surgeons recognized firsthand that many patients with type 2 diabetes undergoing Roux-en-Y gastric bypass (RYGB) experienced resolution of their type 2 diabetes. Pories et al conducted a retrospective singlecohort study of 298 patients with type 2 diabetes or impaired glucose tolerance who underwent RYGB (9). Of those patients, 91\% maintained normal fasting glucose and A1C levels, with follow up as long as 14 years in some patients and with only $4 \%$ attrition. Those who did not remitwere older ( 48 years vs. 40.7 years) and had known type 2 diabetes for longer durations (4.6 years vs. 1.6 years).

The rate of remission of type 2 diabetes after BS varies depending on the procedure; socalled malabsorptive procedures, such as RYGB and biliary pancreatic diversion (BPD), were shown to be more effective than strictly restrictive procedures, such as vertical sleeve gastrectomy (VSG) or adjustable gastric banding (AGB). A meta-analysis of 621 studies published between 1990 and 2006, including 135,246 patients undergoing AGB, gastroplasty, RYGB or BPD, showed that $78.1 \%$ of subjects experienced resolution of type 2 diabetes, and $86.6 \%$ had improvement or resolution after surgery (10). The length of follow up after surgery varied among studies from 1 year to 15 years. Unfortunately, most studies were of short duration and often reported data after fewer than 2 years of follow up. When stratified by procedure, BPD resulted in type 2 diabetes resolution in $95.1 \%$ of patients, RYGB in $80.3 \%$, gastroplasty in $79.7 \%$ and AGB in $56.7 \% 1$ year after surgery (10).

Schauer et al conducted a single-centre prospective trial to investigate clinical parameters associated with type 2 diabetes improvement in 191 individuals after RYGB (11). With a mean follow up of 19.7 months, $83 \%$ of patients saw remission of type 2 diabetes, and the other $17 \%$ saw significant improvement in glucose control. Improvement of type 2 diabetes was defined as fasting blood glucose levels being decreased by $1.38 \mathrm{mmol} / \mathrm{L}(25 \mathrm{mg} / \mathrm{dL})$, A1C levels being decreased by more than $1 \%$ and/or significant reductions in usage of medications for type 2 diabetes (either by discontinuing 1 agent or by reducing the dosage by one half). Resolution of type 2 diabetes, defined as fasting glucose levels below 6.1 $\mathrm{mmol} / \mathrm{L}$ and $\mathrm{A} 1 \mathrm{C}$ levels below $6 \%$ and no longer taking type 2 diabetes medications, was less likely in those with longer durations of type 2 diabetes (10 years vs. 5 years) and with use of insulin prior to surgery (11).

During the past few years, many high-quality randomized controlled trials (RCTs) have compared differing types of BS to intensive or conventional medical therapy (MT). In the Surgical Treatment and Medications Potentially Eradicate Diabetes Efficiently 
(STAMPEDE) trial, patients were randomized to RYGB with MT, VSG with MT or intensive MT alone (2). Intensive MT consisted of an algorithm of metformin, thiazolidinedione, glucagon-like peptide-1 (GLP-1) agonist and insulin, along with lifestyle counselling, weight management and frequent home glucose monitoring. The MT group was supervised every 3 months at a single study centre by an experienced diabetologist. The primary endpoint was defined as A1C levels below $6.0 \%$, with or without the use of medications at the time of evaluation. With 50 subjects in each arm, the primary endpoint was achieved in $12 \%$ of participants in the MT group, $42 \%$ in the RYGB and MT group, and $37 \%$ in the VSG and MT group at 1 year. The remitters after RYGB were not taking medications for type 2 diabetes, but one-third of the remitters in the VSG group required glucose-lowering drugs. It is to be noted that the study population had relatively advanced type 2 diabetes, with average durations longer than 8 years and A1C levels above 9\%, and nearly a quarter of the study participants were taking insulin at the time of enrolment. The relatively low remission rate at 1 year, compared to that found in other studies, highlights the roles of duration and control of type 2 diabetes as being important determinants of remission $(9,11)$.

Mingrone et al conducted a single-centre nonblinded RCT comparing type 2 diabetes remission in patients undergoing RYGB, BPD or conventional MT, including 20 patients in each arm (5). Conventional MT was administered by a multidisciplinary team that included a diabetologist and involved oral hypoglycemic agents and insulin along with diet and lifestyle modification. At 2 years, remission of type 2 diabetes was observed in $95 \%$ of patients after BPD, 75\% after RYGB and 0 in the MT arm. In this study, no preoperative characteristics predicted the likelihood of remission.

Dixon et al compared AGB to conventional MT and showed remission of type 2 diabetes in $73 \%$ of surgical patients and in only 4\% among MT groups at 2-year follow up (4).

Conventional MT was adjusted on a case-by-case basis by an experienced diabetologist at regularly scheduled intervals. The remarkable remission rate after AGB in that study can be explained by the selection criteria for participants who had well controlled type 2 diabetes of short duration, were minimally treated and had lower BMIs, and by the expertise in band adjustment of the Australian team that resulted in $20 \%$ total weight loss at 2 years, compared to $1.4 \%$ weight loss in the MT treatment group (4). In another RCT, Ikramuddin et al compared RYGB to intensive MT in individuals with BMIs between 30.0 and $39.9 \mathrm{~kg} / \mathrm{m}^{2}$ and showed type 2 diabetes remission rates at 1 year to be $75 \%$ and $32 \%$ in the surgical and MT groups, respectively (12). The algorithm for the intensive MT included lifestyle and diet modifications and medications in the following order: metformin, GLP-1 agonist or dipeptidyl peptidase 4 (DPP4) inhibitor, sulfonylurea or pioglitaozone and insulin. In a prospective study, Pournaras et al compared RYGB and AGB in 34 patients and found the remission rate at 3 years to be $72 \%$ after RYGB and only $17 \%$ after AGB (13).

Although many prospective studies have been designed to assess short-term remission rates in those with type 2 diabetes, the observational Swedish Obese Subjects (SOS) study provides data concerning long-term outcomes in those with type 2 diabetes, up to 15 years after intervention (14). In a subgroup of patients with type 2 diabetes studied in this prospective, matched cohort study, 343 subjects underwent BS, and 260 had usual medical 
care consisting of conventional treatment for type 2 diabetes and obesity at primary healthcare centres. Remission of type 2 diabetes in the surgical group was $72.3 \%, 38.1 \%$ and $30.4 \%$ at 2,10 and 15 years, respectively. In the control group, remission of type 2 diabetes was $16.4 \%$ after 2 years and only $6.5 \%$ years after 15 years (14).

Overall, these studies show the superiority of BS over MT as seen in Table $1(2,13)$. However, intensive MT can achieve excellent diabetes control and up to $32 \%$ remission (12). Two large RCTs involving thousands of patients have shown that intensive MT for 5 to 6 years, resulting in A1C levels of $6.5 \%$ to $6.9 \%$, was able to reduce microvascular complications $(15,16)$. Furthermore, MT is constantly evolving. Newer potent agents, such as long-acting GLP-1 agonists and sodium-glucose transporter 2 (SGLT2) inhibitors, which have not been studied in the bariatric/medical therapy trials, may add additional benefits for those with obesity and type 2 diabetes.

\section{Determinants and mechanisms of diabetes remission}

Overall, BS leads to remarkable type 2 diabetes remission in $40 \%$ to $80 \%$ of cases at 1 year $(2,4,5,9-13,17)$. The determinants of early remission include clinical markers of good islet cell function at the time of surgery (i.e. shorter type 2 diabetes duration, lower baseline A1C levels and not using insulin) in addition to the amount of weight loss and the type of surgery (BPD>RYGB $>$ VSG $>$ AGB) $(2,5,9,11,13,18)$. The greater effect of BPD and RYGB on remission of type 2 diabetes is confounded by the greater weight loss observed after these 2 surgeries compared to VSG and AGB $(2,13)$. The predictors of long-term type 2 diabetes remission of up to 15 years in the SOS included greater amount of weight loss and shorter duration of type 2 diabetes (14).

BS results in great, often sustained weight loss, which leads to improvements in blood glucose as well as blood pressure and lipid levels (19). The rate of type 2 diabetes remission tracks the amount of weight loss. Acute caloric restriction leads to improvement in hepatic insulin sensitivity, estimated by homeostatic model assessment of insulin resistance (HOMA-IR) as early as 1 week after RYGB (20). Greater weight loss months after RYGB results in improvement in peripheral insulin sensitivity, best measured by hyperinsulinemiceuglycemic clamp (21). However, improvements in glycemia after RYGB occurs before significant weight loss (11), suggesting that there may be other contributing factors.

The role of the incretins has been suggested as a mechanism of glucose control after RYGB. Incretins are gut hormones that promote insulin secretion, decrease gastric emptying and promote satiety (22). RYGB increases GLP-1 and glucose-dependent insulinotropic polypeptide (GIP) levels as early as 1 month postoperatively (23). VSG, but not AGB (24), initially thought to be merely restrictive, enhances postprandial plasma GLP-1, similar to that of RYGB, likely in relation to accelerated gastrointestinal transit (17).

Studies comparing RYGB and diet-matched weight loss showed superiority of RYGB in raising levels of incretins and decreasing postprandial glucose (25). Blocking the GLP-1 effect with the GLP-1 receptor antagonist exendin $9-39$, infused at $900 \mathrm{pmol} / \mathrm{kg} / \mathrm{min}$, led to worsening of glucose tolerance, decreased beta-cell glucose sensitivity and increased glucagon levels in subjects ( $n=9) 3$ months after RYGB (26). In another study of 9 patients 
with postprandial neuroglycopenia 3.9 years after RYGB, the infusion of exending-39 at 750 $\mathrm{pmol} / \mathrm{kg} / \mathrm{min}$ decreased meal-related insulin release by about $50 \%$ and prevented postprandial hypoglycemia (27). These findings demonstrate a clear role of meal-related enhanced GLP-1 after RYGB in controlling insulin secretion and postprandial glucose.

However, the effect of GLP-1 as the primary mediator of improved glycemic control after RYGB may be limited. Jiménez et al studied postprandial glucose after a meal challenge in 9 individuals with type 2 diabetes remission at least 2 years after RYGB and 8 agematched controls who had not undergone surgery. The infusion of exendin ${ }_{9-39}$ at $750 \mathrm{pmol} / \mathrm{kg} / \mathrm{min}$ resulted in a small, albeit significant, rise in postprandial glucose compared to saline infusion in both the surgical and the control group (28), in spite of a reduction in insulin levels in RYGB subjects with exendin 9-39. Similarly, Vetter el al found that the effects on glucose levels of exendin infusion at $750 \mathrm{pmol} / \mathrm{kg} / \mathrm{min}$ were similar in subjects 4 months after RYGB and in controls who had not undergone surgery but who underwent the same short-termweight reduction through intensive lifestyle changes (29). Shah et al studied 12 subjects without diabetes 5 years after RYGB and 8 nonsurgical controls with meal tests using saline or exendin ${ }_{9-39}$ at $300 \mathrm{pmol} / \mathrm{kg} / \mathrm{min}$. With exendin ${ }_{9-39}$, RYGB patients had decreased insulin levels and elevated postprandial glucose levels, while control subjects experienced elevated postprandial glucose levels and elevated insulin levels. This suggests that GLP-1 antagonism impairs $\beta$ cell function, as measured by the disposition index, in the control subjects but not in individuals after RYGB (30). Collectively, these studies with exendin $_{9-39}$ confirm that GLP-1 plays a predominant role in postprandial insulin secretion with a relative modest effect on glucose. In addition, there seems to be little relationship between GLP-1 release and diabetes status after RYGB (17). More research is needed to determine the extent to which manipulation of the gastrointestinal tract through RYGB or VSG, and the enhanced incretin release, play roles in overall glucose control and diabetes remission.

Other mechanisms, such as changes in serum bile acids $(31,32)$ and in microbiota $(33)$ have been described after RYGB and may also contribute to improved glucose metabolism. However, additional studies are needed for better understanding of the mediation effects of bile acids and host/microbial interactions on metabolism after RYGB.

\section{Diabetes relapse}

Observational studies show that the sustainability of the metabolic effects of BS on type 2 diabetes is highly variable. Type 2 diabetes recurs 3 to 15 years after surgery in $30 \%$ to 50\% of patients who initially experienced remission $(19,34)$. Using retrospective electronic chart analyses of 4434 patients, Arterburn et al showed that type 2 diabetes remission, defined as lack of use of diabetes medications, A1C levels below $6.0 \%$ or fasting blood glucose levels below $5.56 \mathrm{mmol} / \mathrm{L}$, occurred in $68 \%$ of subjects within 5 years of the surgery (34). Of the 2254 patients who experienced type 2 diabetes remission, $35 \%$ relapsed within 5 years of initial remission, with a median time to relapse after surgery of 8.3 years. Factors associated with relapse included preoperative $\mathrm{A} 1 \mathrm{C}$ levels above $6.5 \%$, prior insulin use and longer duration of type 2 diabetes. Preoperative BMIs did not predict type 2 diabetes remission or relapse and, interestingly, weight regain after RYGB was not a predictor of relapse. In a 
small retrospective study of 42 patients undergoing RYGB, DiGiorgi et al showed that of the $64 \%$ of subjects who had complete remission at 6 months, $26 \%$ experienced recurrence of type 2 diabetes after follow up for 3 years (35). The group with recurrence had lower preoperative BMIs ( $47.7 \mathrm{vs.} 52.5 \mathrm{~kg} / \mathrm{m}^{2}$ ), less weight loss and greater weight regain. In a retrospective analysis, Chikunguwo et al studied a larger cohort of 378 subjects with type 2 diabetes who underwent RYGB (18); only 177 subjects were available for at least 5-year follow up (mean follow up, 8.6 years; range, 5 to 16 years). More than $88 \%$ of subjects experienced type 2 diabetes remission at any 1 point. Those who remitted had greater percentages of excess weight loss. However, $48 \%$ of these remitters developed recurrence of type 2 diabetes, and predictors of relapse included female gender, insulin use preoperatively and postsurgical weight regain.

Mingrone et al studied the durability of the effects of BPD, RYGB and conventional MT in 60 patients randomized to 1 of the 3 interventions (36). At 5 years, type 2 diabetes remission was present only in $37 \%$ of patients after RYGB, in $63 \%$ after BPD and in none of the medically treated patients. About $44 \%$ of the surgical patients who remitted after 2 years relapsed by year 5 . However, despite the relapse, $80 \%$ of the surgical patients were able to maintain A1C levels below 7.0\% through diet or metformin alone, suggesting good type 2 diabetes control with minimal therapy (36). In this study, weight changes did not predict type 2 diabetes remission or relapse after surgery.

Similarly, investigators in the STAMPEDE trial further examined the durability of type 2 diabetes remission in a 3-year follow-up study that included $91 \%$ retention of the original cohort (6). At 3 years, the primary endpoint, having A1C levels below $6.0 \%$ while not taking medications, was maintained in 5\%, 38\% and $24 \%$ of patients from the MT, RYGB and VSG groups, respectively. Of the type 2 diabetes remitters in the MT group, $80 \%$ relapsed within the next 2 years, whereas only 24\% relapsed in the RYGB group and 50\% in the VSG group. Despite the high relapse rate in all 3 groups, subjects in RYGB and VSG group used fewer medications than those in the MT group.

Overall, these small- and larger-scale studies as seen in Table 1 show a high rate of type 2 diabetes relapse after BS. The risk factors for relapse of type 2 diabetes after BS are indicators of low betacell function: longer duration of type 2 diabetes, insulin use, poor diabetes control and higher preoperative A1C levels $(18,34)$. In addition, less weight loss after surgery and/or greater weight regain have been associated with diabetes relapse in some, but not all studies $(18,35,36)$. Given the progressive nature of type 2 diabetes and its increased rate with aging, it is not entirely surprising that diabetes relapse occurs after BS. By inducing sustained weight loss, these procedures significantly improve diabetes control and appear to alter the trajectory of the disease without resulting in a permanent cure.

Despite significant relapse rates, type 2 diabetes after BS seems to return in a less severe form, and patients require less insulin and fewer oral medications to achieve control. Further, those with type 2 diabetes who underwent BS experienced the benefit of reduced microvascular outcomes, although long-term data on large number of patients are limited (37). Long-term outcome studies of up to 6.9 years have shown BS to be associated not only with improved glucose control but also with reduced rates of hypertension, hyperlipidemia 
and cardiovascular mortality $(38,39)$. Type 2 diabetes represents a huge burden for patients, so the remission of type 2 diabetes, even for a limited time, may contribute to the sustained increase in quality of life observed after surgery (40).

\section{Risks and Benefits of Bariatric Surgery}

Although BS can have numerous benefits, all surgical procedures and medical treatments are associated with risks. The rigorous preparation of patients, the development of centres of excellence and the elective nature of BS, now performed almost laparascopically, have reduced mortality and complications. Short-term 30-day postoperative mortality and longer term mortality associated with the surgical procedure is very low, less than $1 \%$ for all procedures and a bit higher for RYGB than for VSG or LAGB (41). The frequency of acute surgical complications (bleeding, stomal stenosis, leak, vomiting and electrolyte abnormalities) differ with each procedure: AGB, 7.8\%; VSG, 8.9\%; RYGB, 12\% (41).

Dumping syndrome, with or without reactive hypoglycemia, self-reported in about $10 \%$ of patients 4 to 5 years after RYGB (42) and/or gastroesophogeal reflux after VSG (43) can be invalidating. BS increases the risk for long-term vitamin $\left(\mathrm{B}_{1}, \mathrm{~B}_{12}, \mathrm{C}, \mathrm{A}\right.$ and $\left.\mathrm{D}\right)$, mineral (zinc, copper, iron) or protein deficiencies, which can lead to new comorbidities, such as iron deficiency anemia or secondary hyperparathyroidism, and require lifelong monitoring, prevention and treatment (44). Restrictive procedures, such as AGB and VSG, lead to fewer nutritional deficiencies than malabsorptive procedures such as RYGB (43). Laboratory testing for these deficiencies should be performed at regular intervals and at least annually for life (45).

Although the upfront cost of BS is high, downstream cost savings are achieved through decreased comorbidities and decreased use of costly prescription drugs, physician visit costs and hospital costs. One study found that the savings within 2 years after laparoscopic procedures and 4 years after open procedures offset initial costs (46); although, other studies have not shown similar long-term cost savings $(47,48)$. In a 6-year study comparing healthcare costs in surgically treated and medically treated patients with obesity, Weiner et al found increased overall healthcare costs in the first 3 years after surgery but similar costs in later years (49). Authors found that the initial BS procedure cost ranges from $\$ 15,000$ to $\$ 30,000$ (in 2005 US dollars), and prescription drug costs were reduced by $20 \%$ to $30 \%$ in the 3 years after BS (49). BS has been shown to improve quality of life, increase longevity and reduce disability and work absenteeism, parameters harder to account for in cost analysis. Cost analyses often do not take into account the possible occurrence of long-term mineral, vitamin and/or protein deficiencies, with or without associated comorbidities, and the associated costs of treating diabetes relapse. Although BS can be considered costeffective in terms of reducing obesity-associated comorbidities, the question of long-term (lifetime) cost savings is still a matter of ongoing research.

\section{Screening and Treatment of Diabetes after Surgery}

A significant number of patients experience either persistent type 2 diabetes after surgery or, after a few months or years in remission, experience relapse in the years following their 
surgery. The distinction between persistent and relapsing type 2 diabetes may be important in terms of mechanism. However, the 2 may be hard to distinguish if diabetes status is not documented by longitudinal screening. Buse et al recommended at least a 5 -year continuous state of complete remission before a person with known type 2 diabetes is considered cured (7). Most patients discontinue their treatments for type 2 diabetes and, unless they continue self-monitoring, may not know whether their glucose levels have normalized. It becomes, therefore, imperative for healthcare providers to remain vigilant and continue to monitor glucose control after BS. Patients at high risk for persistent type 2 diabetes and/or relapse should be advised to continue sporadic but regular self-monitoring of fasting blood glucose levels because the postprandial glucose levels may be difficult to interpret after RYGB. An annual A1C-level test should be ordered, at the minimum. However, iron deficiency anemia, common after BS, particularly in premenopausal women, can, for unclear reasons, falsely elevate $\mathrm{A} 1 \mathrm{C}$ levels (50). A1C levels usually decrease after iron supplementation in iron deficiency anemia (51). Therefore, proper assessment of type 2 diabetes status requires close attention to iron levels, particularly in patients with only mildly elevated A1C levels.

The management of type 2 diabetes after BS can represent a challenge. Accelerated food transit after RYGB or VSG may be responsible for dumping syndrome, with or without postprandial hypoglycemia (52). The hypoglycemia occurs usually 2 to 3 hours postprandially, triggered by hyperinsulinism resulting from an exaggerated incretin response (52). Patients will need adequate education prior to surgery regarding altered food transit time, glucose absorption and metabolism and dumping syndrome. The hypoglycemia can lead to overcorrection with ingestion of simple carbohydrates and trigger a vicious circle of alternating low and high blood glucose levels, which are best addressed through dietary management.

The optimal treatment of type 2 diabetes after BS should, ideally, promote weight loss or weight neutrality and avoid hypoglycemia. With that in mind, metformin would be the firstline treatment because it is weight neutral. Some patients may find difficult to swallow metformin, given the size of the pill. The insulin sensitizers, thiazolidinediones, lead to weight gain. Sulfonylureas can lead to hypoglycemia. The use of incretin analogues, such as DPP4 inhibitors or GLP-1 agonists, have not been studied in the context of post-BS, and whether they are safe or efficacious in the context of high GLP-1 levels after RYGB or VSG, for example, requires clinical investigation. SGLT2 inhibitors, newer agents that have not been studied in the bariatric population, may be attractive because they promote weight loss through glycosuria, as long as patients can maintain proper levels of hydration. For patients with recurrence of mild type 2 diabetes with A1C levels below 7\%, some dietary management, self-monitoring and metformin may suffice. For more severe relapse with elevated A1C levels, a basal insulin may be indicated and may be the safest. Prandial insulin may be more difficult to adjust because of the accelerated gastric emptying.

\section{Conclusions}

BS improves glycemia by inducing calorie restriction and sustained weight loss, thus improving insulin sensitivity and $\beta$ cell function. Additionally, some surgeries, such as RYGB and VSG, may have weight-independent effects, possibly via enhancement of the 
incretin system and insulin secretion, particularly during meals. The International Diabetes Federation and the American Diabetes Association now recommend BS as a part of the algorithm in type 2 diabetes management $(53,54)$. Although BS does not provide a longlasting surgical cure for type 2 diabetes, it has a robust and sustained effect on glucose metabolism, until now unmatched by intensive MT. It is unclear what the true prevalence of type 2 diabetes is after BS because attrition is quite significant in this population, and there is a lack of long-term data. The use of electronic medical records may allow future collection of long-term data concerning the long-term benefits of these surgeries in much larger cohorts of patients.

The number of bariatric surgeries used to treat obesity and type 2 diabetes will only rise in the coming years. More studies are needed to test the efficacy and safety of medications for type 2 diabetes so as to define optimal pharmacotherapy for those with type 2 diabetes after BS. It is imperative for patients and healthcare providers to be cognizant of the long-term effects of each surgery on glucose metabolism and the possibility of relapse of type 2 diabetes. This will help patients to adjust their expectations and choose the right surgery. Long-term studies are needed to examine long term effects of BS on type 2 diabetes and its micro- and macrovascular complications.

\section{References}

1. Mokdad AH, Bowman BA, Ford ES, et al. The continuing epidemics of obesity and diabetes in the United States. JAMA. 2001; 286:1195-200. [PubMed: 11559264]

2. Schauer PR, Kashyap SR, Wolski K, et al. Bariatric surgery versus intensive medical therapy in obese patients with diabetes. N Engl J Med. 2012; 366:1567-76. [PubMed: 22449319]

3. Wing RR, Bolin P, et al. Look AHEAD Research Group. Cardiovascular effects of intensive lifestyle intervention in type 2 diabetes. N Engl J Med. 2013; 369:145-54. [PubMed: 23796131]

4. Dixon JB, O'Brien PE, Playfair J, et al. Adjustable gastric banding and conventional therapy for type 2 diabetes: A randomized controlled trial. JAMA. 2008; 299:316-23. [PubMed: 18212316]

5. Mingrone G, Panunzi S, De Gaetano A, et al. Bariatric surgery versus conventional medical therapy for type 2 diabetes. N Engl J Med. 2012; 366:1577-85. [PubMed: 22449317]

6. Schauer PR, Bhatt DL, Kirwan JP, et al. Bariatric surgery versus intensive medical therapy for diabetes: 3-year outcomes. N Engl J Med. 2014; 370:2002-13. [PubMed: 24679060]

7. Buse JB, Caprio S, Cefalu WT, et al. How do we define cure of diabetes? Diabetes Care. 2009; 32:2133-5. [PubMed: 19875608]

8. Schwarz A, Büchler M, Usinger K, et al. Importance of the duodenal passage and pouch volume after total gastrectomy and reconstruction with the Ulm pouch: Prospective randomized clinical study. World J Surg. 1996; 20:60-6. discussion 66-7. [PubMed: 8588415]

9. Pories WJ, Swanson MS, MacDonald KG, et al. Who would have thought it? An operation proves to be the most effective therapy for adult-onset diabetes mellitus. Ann Surg. 1995; 222:339-50. discussion 350-2. [PubMed: 7677463]

10. Buchwald H, Estok R, Fahrbach K, et al. Weight and type 2 diabetes after bariatric surgery: Systematic review and meta-analysis. Am J Med. 2009; 122:248-56, e5. [PubMed: 19272486]

11. Schauer PR, Burguera B, Ikramuddin S, et al. Effect of laparoscopic Roux-en Y gastric bypass on type 2 diabetes mellitus. Ann Surg. 2003; 238:467-84. discussion 84-5. [PubMed: 14530719]

12. Ikramuddin S, Korner J, Lee WJ, et al. Roux-en-Y gastric bypass vs intensive medical management for the control of type 2 diabetes, hypertension, and hyperlipidemia: The Diabetes Surgery Study randomized clinical trial. JAMA. 2013; 309:2240-9. [PubMed: 23736733]

13. Pournaras DJ, Osborne A, Hawkins SC, et al. Remission of type 2 diabetes after gastric bypass and banding: Mechanisms and 2-year outcomes. Ann Surg. 2010; 252:966-71. [PubMed: 21107106] 
14. Sjostrom L, Peltonen M, Jacobson P, et al. Association of bariatric surgery with long-term remission of type 2 diabetes and with microvascular and macrovascular complications. JAMA. 2014; 311:2297-304. [PubMed: 24915261]

15. Duckworth W, Abraira C, Moritz T, et al. Glucose control and vascular complications in veterans with type 2 diabetes. N Engl J Med. 2009; 360:129-39. [PubMed: 19092145]

16. Patel A, MacMahon S, et al. ADVANCE Collaborative Group. Intensive blood glucose control and vascular outcomes in patients with type 2 diabetes. N Engl J Med. 2008; 358:2560-72. [PubMed: 18539916]

17. Jiménez A, Casamitjana R, Flores L, et al. Long-term effects of sleeve gastrectomy and Roux-en-Y gastric bypass surgery on type 2 diabetes mellitus in morbidly obese subjects. Ann Surg. 2012; 256:1023-9. [PubMed: 22968072]

18. Chikunguwo SM, Wolfe LG, Dodson P, et al. Analysis of factors associated with durable remission of diabetes after Roux-en-Y gastric bypass. Surg Obes Relat Dis. 2010; 6:254-9. [PubMed: 20303324]

19. Sjostrom L, Lindroos AK, Peltonen M, et al. Lifestyle, diabetes, and cardiovascular risk factors 10 years after bariatric surgery. N Engl J Med. 2004; 351:2683-93. [PubMed: 15616203]

20. Isbell JM, Tamboli RA, Hansen EN, et al. The importance of caloric restriction in the early improvements in insulin sensitivity after Roux-en-Y gastric bypass surgery. Diabetes Care. 2010; 33:1438-42. [PubMed: 20368410]

21. Bojsen-Moller KN, Dirksen C, Jørgensen NB, et al. Early enhancements of hepatic and later of peripheral insulin sensitivity combined with increased postprandial insulin secretion contribute to improved glycemic control after Rouxen- Y gastric bypass. Diabetes. 2014; 63:1725-37. [PubMed: 24241533]

22. Tasyurek HM, Altunbas HA, Balci MK, Sanlioglu S. Incretins: Their physiology and application in the treatment of diabetes mellitus. Diabetes Metab Res Rev. 2014; 30:354-71. [PubMed: 24989141]

23. Laferrere B, Heshka S, Wang K, et al. Incretin levels and effect are markedly enhanced 1month after Roux-en-Y gastric bypass surgery in obese patients with type 2 diabetes. Diabetes Care. 2007; 30:1709-16. [PubMed: 17416796]

24. Kashyap SR, Daud S, Kelly KR, et al. Acute effects of gastric bypass versus gastric restrictive surgery on beta-cell function and insulinotropic hormones in severely obese patients with type 2 diabetes. Int J Obes (Lond). 2010; 34:462-71. [PubMed: 20029383]

25. Laferrere B, Teixeira J, McGinty J, et al. Effect of weight loss by gastric bypass surgery versus hypocaloric diet on glucose and incretin levels in patients with type 2 diabetes. J Clin Endocrinol Metab. 2008; 93:2479-85. [PubMed: 18430778]

26. Jorgensen NB, Dirksen C, Bojsen-Møller KN, et al. Exaggerated glucagon-like peptide 1 response is important for improved beta-cell function and glucose tolerance after Roux-en-Y gastric bypass in patients with type 2 diabetes. Diabetes. 2013; 62:3044-52. [PubMed: 23649520]

27. Salehi M, Gastaldelli A, D'Alessio DA. Blockade of glucagon-like peptide 1 receptor corrects postprandial hypoglycemia after gastric bypass. Gastroenterology. 2014; 146:669-80, e2. [PubMed: 24315990]

28. Jimenez A, Casamitjana R, Viaplana-Masclans J, et al. GLP-1 action and glucose tolerance in subjects with remission of type 2 diabetes after gastric bypass surgery. Diabetes Care. 2013; 36:2062-9. [PubMed: 23359363]

29. Vetter ML, Wadden TA, Teff KL, et al. GLP-1 plays a limited role in improved glycemia shortly after Roux-en-Y gastric bypass: A comparison with intensive lifestyle modification. Diabetes. 2015; 64:434-46. [PubMed: 25204975]

30. Shah M, Law JH, Micheletto F, et al. Contribution of endogenous glucagon-like peptide 1 to glucose metabolism after Roux-en-Y gastric bypass. Diabetes. 2014; 63:483-93. [PubMed: 24089513]

31. Patti ME, Houten SM, Bianco AC, et al. Serum bile acids are higher in humans with prior gastric bypass: Potential contribution to improved glucose and lipid metabolism. Obesity (Silver Spring). 2009; 17:1671-7. [PubMed: 19360006] 
32. Dutia R, Embrey M, O'Brien CS, et al. Temporal changes in bile acid levels and 12alphahydroxylation after Roux-en-Y gastric bypass surgery in type 2 diabetes. Int J Obes (Lond). 2015; 39:806-13. [PubMed: 25599611]

33. Liou AP, Paziuk M, Luevano JM Jr, et al. Conserved shifts in the gut microbiota due to gastric bypass reduce host weight and adiposity. Sci Transl Med. 2013; 5:178ra41.

34. Arterburn DE, Bogart A, Sherwood NE, et al. A multisite study of long-term remission and relapse of type 2 diabetes mellitus following gastric bypass. Obes Surg. 2013; 23:93-102. [PubMed: 23161525]

35. DiGiorgi M, Rosen DJ, Choi JJ, et al. Re-emergence of diabetes after gastric bypass in patients with mid- to long-term follow-up. Surg Obes Relat Dis. 2010; 6:249-53. [PubMed: 20510288]

36. Mingrone G, Panunzi S, De Gaetano A, et al. Bariatric-metabolic surgery versus conventional medical treatment in obese patients with type 2 diabetes: 5 year follow-up of an open-label, singlecentre, randomised controlled trial. Lancet. 2015; 386:964-73. [PubMed: 26369473]

37. Coleman KJ, Haneuse S, Johnson E, et al. Long-term microvascular disease outcomes in patients with type 2 diabetes after bariatric surgery: Evidence for the legacy effect of surgery. Diabetes Care. 2016; 39:1400-7. [PubMed: 27271192]

38. Arterburn DE, Olsen MK, Smith VA, et al. Association between bariatric surgery and long-term survival. JAMA. 2015; 313:62-70. [PubMed: 25562267]

39. Puzziferri N, Roshek TB 3rd, Mayo HG, et al. Long-term follow-up after bariatric surgery: A systematic review. JAMA. 2014; 312:934-42. [PubMed: 25182102]

40. Andersen JR, Aasprang A, Karlsen TI, et al. Health-related quality of life after bariatric surgery: A systematic review of prospective long-term studies. Surg Obes Relat Dis. 2015; 11:466-73. [PubMed: 25820082]

41. Chang SH, Stoll CR, Song J, et al. The effectiveness and risks of bariatric surgery: An updated systematic review and meta-analysis, 2003-2012. JAMA Surg. 2014; 149:275-87. [PubMed: 24352617]

42. Nielsen JB, Pedersen AM, Gribsholt SB, et al. Prevalence, severity, and predictors of symptoms of dumping and hypoglycemia after Roux-en-Y gastric bypass. Surg Obes Relat Dis. 2016; 12:15628. [PubMed: 27425831]

43. Tack J, Deloose E. Complications of bariatric surgery: Dumping syndrome, reflux and vitamin deficiencies. Best Pract Res Clin Gastroenterol. 2014; 28:741-9. [PubMed: 25194187]

44. Schweitzer DH, Posthuma EF. Prevention of vitamin and mineral deficiencies after bariatric surgery: Evidence and algorithms. Obes Surg. 2008; 18:1485-8. [PubMed: 18369680]

45. Mechanick JI, Youdim A, Jones DB, et al. Clinical practice guidelines for the perioperative nutritional, metabolic, and nonsurgical support of the bariatric surgery patient: 2013 update, cosponsored by American Association of Clinical Endocrinologists, the Obesity Society, and American Society for Metabolic \& Bariatric Surgery. Surg Obes Relat Dis. 2013; 9:159-91. [PubMed: 23537696]

46. Cremieux PY, Buchwald H, Shikora SA, et al. A study on the economic impact of bariatric surgery. Am J Manag Care. 2008; 14:589-96. [PubMed: 18778174]

47. Maciejewski ML, Livingston EH, Smith VA, et al. Health expenditures among highrisk patients after gastric bypass and matched controls. Arch Surg. 2012; 147:633-40. [PubMed: 22802057]

48. Neovius M, Narbro K, Keating C, et al. Health care use during 20 years following bariatric surgery. JAMA. 2012; 308:1132-41. [PubMed: 22990272]

49. Weiner JP, Goodwin SM, Chang HY, et al. Impact of bariatric surgery on health care costs of obese persons: A 6-year follow-up of surgical and comparison cohorts using health plan data. JAMA Surg. 2013; 148:555-62. [PubMed: 23426865]

50. Weng TC, Chang $\mathrm{CH}$, Dong YH, et al. Anaemia and related nutrient deficiencies after Roux-en-Y gastric bypass surgery: A systematic review and metaanalysis. BMJ Open. 2015; 5:e006964.

51. English E, Idris I, Smith G, et al. The effect of anaemia and abnormalities of erythrocyte indices on HbA1c analysis: A systematic review. Diabetologia. 2015; 58:1409-21. [PubMed: 25994072]

52. Ritz P, Vaurs C, Barigou M, Hanaire H. Hypoglycaemia after gastric bypass: Mechanisms and treatment. Diabetes Obes Metab. 2016; 18:217-23. [PubMed: 26508374] 
53. Dixon JB, Zimmet P, Alberti KG, et al. Bariatric surgery: An IDF statement for obese type 2 diabetes. Diabet Med. 2011; 28:628-42. [PubMed: 21480973]

54. American Diabetes Association. Executive summary: Standards of medical care in diabetes, 2012. Diabetes Care. 2012; 35(Suppl. 1):S4-10. [PubMed: 22187471] 


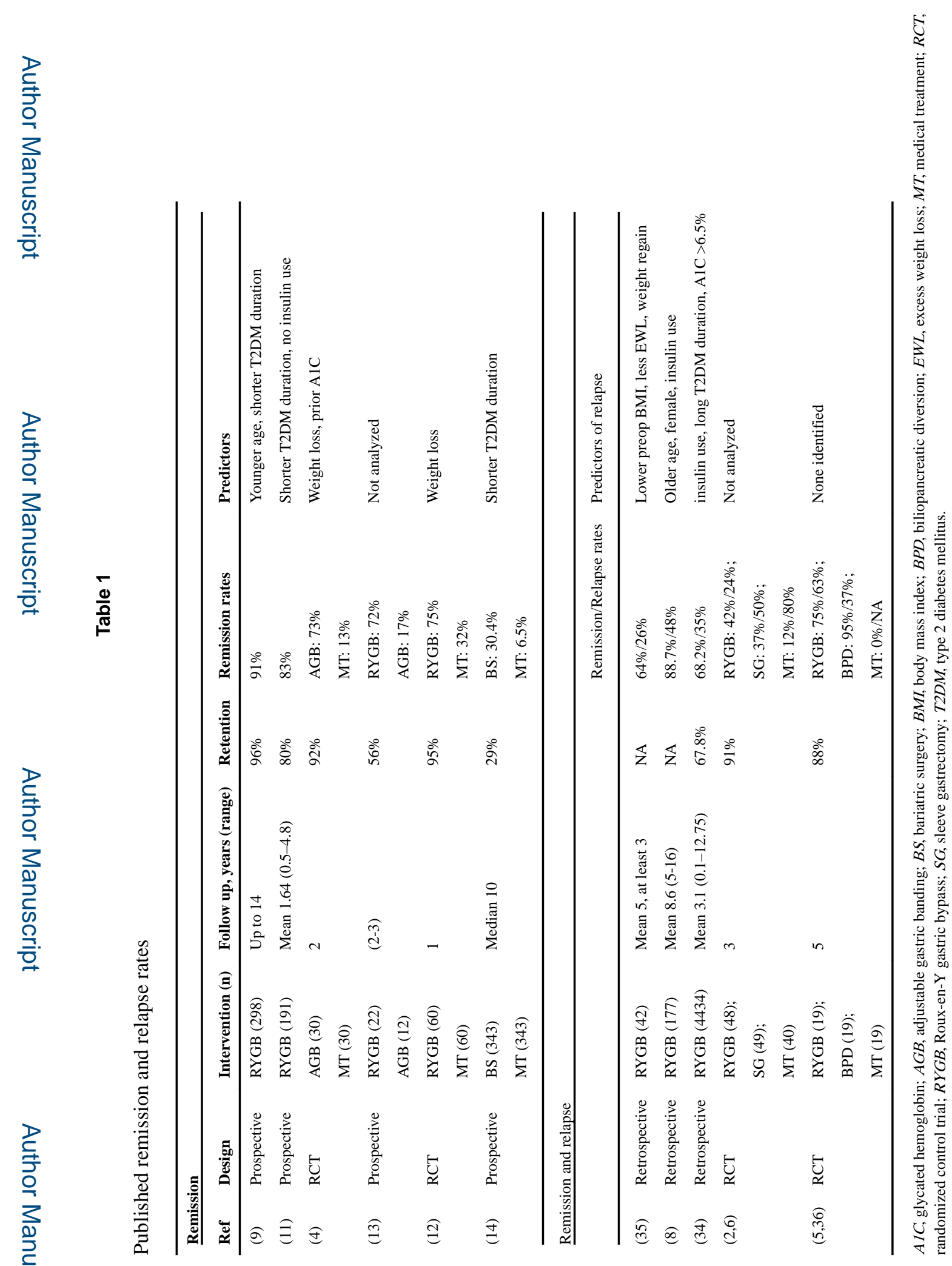

? 